\title{
On the Rings of Differential Operators
}

\section{Ammar S. Mahmood \\ College of Education \\ University of Mosul, Iraq}

\section{Received on: 06/06/2005}

\section{ABSTRACT}

Accepted on: 26/12/2005

The rings of differential operators have been studied by many mathematicians like Musson [5], Smith and Stafford [7]. Jones in [2] and [3] introduced new ideas for such kind of rings and he found a new line.

In this work, we generalize many of the relations of Jones in the first part, and we found a new proof for some relations of Jones.

Keywords: rings of differential operators.

$$
\begin{aligned}
& \text { حول الحلقات من النوع التفاضلي } \\
& \text { عمار صديق محمود } \\
& \text { كلية التربية، جامعة الدوصل } \\
& \text { تاريخ القبول: 2005/12/26 } \\
& \text { تاريخ الاستلام: 2005/06/06 } \\
& \text { الملخص } \\
& \text { درسن الحلقات من النوع التفاضلي المميز الكثيرُ من الباحثين ولعل من أبرزهم مُسن في }
\end{aligned}
$$

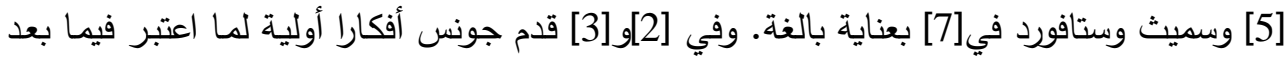

$$
\begin{aligned}
& \text { بأنه بداية لخط جديد من هذا النوع من الحلقات. حاولنا في هذا البحث تعميم الكثير من علاقات } \\
& \text { جونس في الجانب الأول وفي الجانب الثاني تمكنا من ايجاد براهين مختلفة لقسم من هذه العلاقات } \\
& \text { التي أوجدها جونس في [2] و[3]. }
\end{aligned}
$$

\section{Introduction:}

Let $\mathrm{k}$ be an algebraically closed field of characteristic zero. For a commutative k-algebra $\mathrm{A}$, we defined $\mathrm{D}(\mathrm{A}):=\bigcup_{\mathrm{i}=0}^{\infty} \mathrm{D}^{\mathrm{i}}(\mathrm{A})$ where $\mathrm{D}^{0}(\mathrm{~A})=\operatorname{End}_{\mathrm{A}}(\mathrm{A})$ and $\mathrm{D}^{\mathrm{i}}(\mathrm{A})=\left\{\theta \in \operatorname{End}_{\mathrm{k}}(\mathrm{A}):[\theta, \mathrm{a}] \in \mathrm{D}^{\mathrm{i}-1}(\mathrm{~A}), \forall \mathrm{a} \in \mathrm{A}\right\}$. Then $\mathrm{D}(\mathrm{A})$ is a sub ring of $\operatorname{End}_{\mathrm{k}}(\mathrm{A})$, called the ring of differential operators on $\mathrm{A}$. For an irreducible affine variety $\mathrm{X}$, we define $\mathrm{D}_{1}(\mathrm{x})$ := $\mathrm{D}(\mathrm{O}(\mathrm{x}))$ where $\mathrm{O}(\mathrm{x})$ is a ring of regular functions of $\mathrm{X}$ and call this ring $\mathrm{D}(\mathrm{O}(\mathrm{x}))$ of differential operators on $\mathrm{X}$.

Let $\mathrm{N}$ be a free Z-module of rank $\mathrm{r}$ and $\mathrm{M}=\operatorname{Hom}_{\mathrm{z}}(\mathrm{N}, \mathrm{Z})$ its $\mathrm{Z}$ module dual. Then we have a bilinear pairing $\langle\rangle:, \mathrm{M} \times \mathrm{N} \rightarrow \mathrm{Z}$ which 
extends to $\langle\rangle:, \mathrm{M}_{\mathrm{Q}} \times \mathrm{N}_{\mathrm{Q}} \rightarrow \mathrm{Q}$ where $\mathrm{M}_{\mathrm{Q}}=\mathrm{M} \otimes_{\mathrm{Z}} \mathrm{Q}$ and $\mathrm{N}_{\mathrm{Q}}=\mathrm{N} \otimes_{\mathrm{z}} \mathrm{Q}$. Let $\mathrm{f} \in\left(\mathrm{M}_{\mathrm{Q}}\right)^{*}$ be a subset of the form $\mathrm{H}_{\mathrm{f}}=\left\{\lambda \in \mathrm{M}_{\mathrm{Q}}: \mathrm{f}(\lambda) \geq 0\right\}$, defined is a half space of $\mathrm{M}_{\mathrm{Q}}$. Let $\mathrm{V}$ be a finite dimensional vector space over $\mathrm{Q}$.

A subset $\{\mathrm{v} \in \mathrm{V}: \lambda(\mathrm{v}) \geq 0\}$ where $\lambda: \mathrm{V} \rightarrow \mathrm{Q}$ is a non-zero linear function called a half space of $\mathrm{V}$. A cone $\sigma$, in $\mathrm{V}$ is the intersection of a finite number of half spaces. It can be written in the form:

$$
\sigma=\sum_{\mathrm{i}=1}^{\mathrm{s}} \mathrm{Q}_{\geq 0} \mathrm{v}_{\mathrm{i}} \quad \text { for } \mathrm{v}_{1}, \mathrm{v}_{2}, \ldots \ldots ., \mathrm{v}_{\mathrm{s}} \in \mathrm{V} .
$$

A subset of $\sigma$ of the form $\{\mathrm{v} \in \mathrm{V}: \lambda(\mathrm{v})=0\}$ where $\lambda: \mathrm{V} \rightarrow \mathrm{Q}$ is a linear functional positive on $\sigma$ is called a face of $\sigma$. The dimension of the cone $\sigma$ is the dimension of the vector space $\sigma-\sigma$ over $\mathrm{Q}$.

Consider a cone $\sigma$ in $\mathrm{N}_{\mathrm{Q}}$. Let $\stackrel{\mathrm{v}}{\sigma}=\left\{\mathrm{p} \in \mathrm{M}_{\mathrm{Q}}:\langle\mathrm{p}, \sigma\rangle \subseteq \mathrm{Q}_{\geq 0}\right\}$. Then $\sigma$ is an $\mathrm{r}$-dimensional cone in $\mathrm{M}_{\mathrm{Q}}$ and $\sigma \mathrm{v} \cap \mathrm{M}$ is finitely generated additive

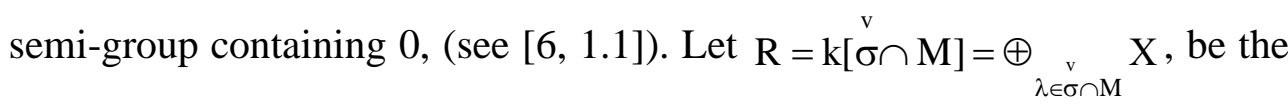
semi-group algebra.

Here $\mathrm{X}^{\lambda}$ is a formal monomial and the multiplication is given by the semi-group addition. Choose once and for all a $\mathrm{Z}$-basis of $\mathrm{M}$, say $\left\{\mathrm{m}_{1}, \mathrm{~m}_{2}, \ldots \ldots \ldots \mathrm{m}_{\mathrm{r}}\right\}$. Then set $\mathrm{x}_{\mathrm{i}}=\mathrm{X}^{\mathrm{m}_{\mathrm{i}}}$ and $\partial_{\mathrm{i}}=\partial / \partial \mathrm{x}_{\mathrm{i}}$.

Jones in [3] starts with a single relation. In this paper, we use that relation to obtain a new general basis.

\section{The semi-groups $\Lambda$ and $\underline{\tilde{\Lambda}}$}

In this section, we repeat in the first part the definition of the semi-group $\Lambda$, we give many of its features. Jones in [3] used these features to define another semi-group, denoted by $\widetilde{\Lambda}$.

Consider a finite set $F_{1}$ of hyper-planes such that each is parallel but

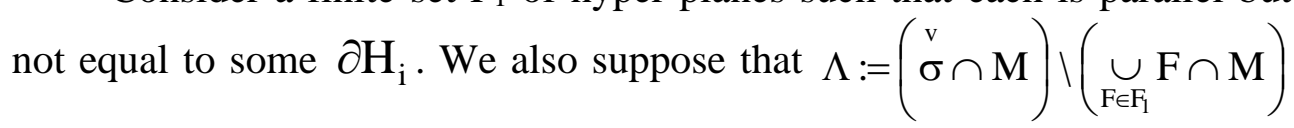
is a semi-group. We call such a semi-group a hyper-plane deleted sub semigroup of $\left(\begin{array}{l}\mathrm{v} \\ \sigma\end{array} \mathrm{M}\right)$. For any hyper-plane $\mathrm{F}$ in $\mathrm{M}_{\mathrm{Q}}$, let $\mathrm{e}(\mathrm{F})$ be the polynomial 
of $\mathrm{S}\left(\mathrm{M}_{\mathrm{Q}}^{*}\right)$ which defines $\mathrm{F}$. Note that for $\mathrm{F} \in \mathrm{F}_{1}, \mathrm{e}(\mathrm{F})$ are the polynomials of degree 1, with rational coefficients.

Definition 2.1: A semi-group $\Gamma$ is said to be normal if one of the following equivalents conditions holds:

(1) For $a, b, c \in \Gamma$ if $a+n b=n c$ for $n \in N$, then $a=n a_{1}$ for some $\mathrm{a}_{1} \in \Gamma$.

(2) For $a \in Z \Gamma$ and $0 \neq n \in N$, if $n a \in \Gamma$, then $a \in \Gamma$.

For an arbitrary finitely generated semi-group the normalization of $\Gamma, \tilde{\Gamma}$ is defined as: $\tilde{\Gamma}=\{\mathrm{a} \in \mathrm{Z} \Gamma: \mathrm{na} \in \Gamma$, for some $0 \neq \mathrm{n} \in \mathrm{N}\}$.

Musson in [4,1.3] introduced the following proposition:

Proposition 2.2: For a semi-group $\Lambda$, the following are equivalents:

(1) $\Lambda$ is normal;

(2) For any field $\mathrm{k}, \mathrm{k} \Lambda$ is an integrally closed Noetherian domain;

(3) For some $0 \leq \mathrm{t} \leq \mathrm{s}, \Lambda$ is isomorphic to a semi-group of the form $\left(\mathrm{Z}_{\geq 0}^{\mathrm{t}} \times \mathrm{Z}^{\mathrm{s}-\mathrm{t}}\right) \cap \mathrm{V}$, where $\mathrm{V}$ is a subspace of $\mathrm{M}_{\mathrm{Q}}$ and $\operatorname{dim}$ $\mathrm{Q} \Lambda=\mathrm{r}$;

(4) For some $t, n \geq 0, \Lambda$ is isomorphic to a semi-group of the form $\mathrm{M} \cap \mathrm{H}_{1} \cap \ldots \cap \mathrm{H}_{\mathrm{t}}$ where $\mathrm{H}_{\mathrm{i}}$ are half spaces in $\mathrm{M}_{\mathrm{Q}}$ and $\mathrm{Z} \Lambda=\mathrm{M}$.

In the following lemma, Jones in [3] defined another semi-group $\tilde{\Lambda}$ :-

\section{Lemma 2.3:}

(1) $\tilde{\Lambda}:=\stackrel{v}{\sigma} \cap M$;

(2) $\mathrm{k} \Lambda=\mathrm{k}[\mathrm{v} \sigma \mathrm{\sigma}]=\mathrm{k} \tilde{\Lambda}$.

\section{Proof:}

(1) For all $\lambda \in \sigma \cap \mathrm{v}$ and $\mathrm{a} \in \mathrm{N}$, we have $\mathrm{a} \lambda \in \Lambda$ because $\Lambda$ is a semi-group of $\sigma \cap M$ such that $\otimes_{\geq 0} \Lambda=\sigma$ and $Z \Lambda=M$, then $\mathrm{M}=\mathrm{Z \Lambda}=\mathrm{Z} \tilde{\Lambda} \quad$ and $\quad \mathrm{M}=(\sigma \cap \mathrm{v} \cap)$ therefore $\mathrm{\sigma} \cap \mathrm{M}=\tilde{\Lambda}$.

(2) The second result is true by the proposition (2.2) in the second part 
Now, For $\Lambda$ an arbitrary semi-group such that $Z \Lambda=M$, let $\mathrm{k} \Lambda$ be the associated semi-group algebra of $\Lambda$ and $\mathrm{D}(\mathrm{k} \Lambda)$ the ring of differential operators. Then $\mathrm{D}(\mathrm{k} \Lambda) \subseteq \mathrm{D}(\mathrm{kM})$

$$
\begin{aligned}
& =\mathrm{D}\left(\mathrm{k}\left[\mathrm{x}_{1}^{ \pm 1}, \ldots \ldots ., \mathrm{x}_{\mathrm{r}}^{ \pm 1}\right]\right. \\
& =\mathrm{k}\left[\mathrm{x}_{1}^{ \pm 1}, \ldots \ldots, \mathrm{x}_{\mathrm{r}}^{ \pm 1}, \partial_{1}, \ldots \ldots, \partial_{\mathrm{r}}\right] .
\end{aligned}
$$

Clearly $x_{i} \partial_{i}$ is in $D(k \Lambda)$. Now for $\mu \in Z \Lambda, \quad x_{i} \partial_{i} * x^{\mu}=\mu_{i} x^{\mu}$, where $\mu=\left(\mu_{1}, \mu_{2}, \ldots, \mu_{r}\right)$.

Let $\mathrm{W}=\mathrm{Q}\left[\mathrm{x}_{1} \partial_{1}, \ldots \ldots . \mathrm{x}_{\mathrm{r}} \partial_{\mathrm{r}}\right] \otimes_{\mathrm{Q}} \mathrm{k}$ then $\mathrm{W} \subseteq \mathrm{D}(\mathrm{k} \Lambda)$. Thus the elements of $\mathrm{W}$ define polynomial functions from $\mathrm{Z} \Lambda \subseteq \mathrm{M}_{\mathrm{Q}}$ to $\mathrm{k}$ by the rule $\mathrm{x}_{\mathrm{i}} \partial_{\mathrm{i}}(\mu)=\mu_{\mathrm{i}}$ for $\mu \in \mathrm{Z} \Lambda$.

Thus for $\mathrm{f} \in \mathrm{W}$ and $\mu \in \mathrm{Z} \Lambda, \mathrm{f}\left(\mathrm{x}_{1} \partial_{1}, \ldots, \mathrm{x}_{\mathrm{r}} \partial_{\mathrm{r}}\right) \mathrm{x}^{\mu}=\mathrm{f}(\mu) \mathrm{x}^{\mu}$.

Definition 2.4: For $A, B \subseteq M$ and $\lambda \in M$, define $\Omega_{\mathrm{A}, \mathrm{B}}(\lambda)=\{\mu \in \mathrm{A} \vdots \lambda+\mu \notin \mathrm{B}\}$

Also let

$\Omega_{\mathrm{A}}(\lambda)=\Omega_{\mathrm{A}, \mathrm{A}}(\lambda)$. For $\Omega \subseteq$ M define $\bar{\Omega}$ to be the Zariski closure in $\mathrm{M}_{\mathrm{Q}}$ and let $\mathrm{I}(\Omega)=\{\mathrm{f} \in \mathrm{W}: \mathrm{f}(\rho)=0, \forall \rho \in \Omega\}$. Also $\mathrm{I}(\Omega)=\mathrm{I}(\bar{\Omega})$.

Lemma 2.5: [Musson]

Let $\Lambda$ be a semi-group of $\mathrm{M}$ with $\mathrm{Q} \Lambda=\mathrm{M}_{\mathrm{Q}}$. For $\mathrm{g} \in\left(\mathrm{M}_{\mathrm{Q}}\right)^{*}$ and $\mathrm{b} \in \mathrm{Q}$ set $\Lambda_{\mathrm{b}}=\{\lambda \in \Lambda: \mathrm{g}(\lambda)=\mathrm{b}\}$. Suppose that:

(1) $\Lambda_{\mathrm{b}} \neq \phi$;

(2) $\operatorname{dim}_{\mathrm{Q}} \mathrm{Q} \Lambda_{0}=\mathrm{r}-1$. Then $\bar{\Lambda}_{\mathrm{b}}=\left\{\lambda \in \mathrm{M}_{\mathrm{Q}} \vdots \mathrm{g}(\lambda)=\mathrm{b}\right\}$

Now, we decompose $\Omega_{\tilde{\Lambda}}(\lambda)$ into pieces, $\Omega_{\tilde{\Lambda}}^{\mathrm{i}}(\lambda)=\left\{\mu \in \tilde{\Lambda}: \lambda+\mu \notin \mathrm{H}_{\mathrm{i}} \cap \mathrm{M}\right\}$.

Observer that $\Omega_{\widetilde{\Lambda}}(\lambda)=\cup \Omega_{\widetilde{\Lambda}}^{\mathrm{i}}(\lambda)$. 


\section{Proposition2.6:}

$\overline{\Omega_{\tilde{\Lambda}}^{\mathrm{i}}(\lambda)}=\left\{\mu \in \mathrm{M}_{\mathrm{Q}} \vdots \mathrm{h}_{\mathrm{i}}(\mu) \in \mathrm{h}_{\mathrm{i}}(\mathrm{M}) \& 0 \leq \mathrm{h}_{\mathrm{i}}(\mu)<-\mathrm{h}_{\mathrm{i}}(\lambda)\right\}$, for $\mathrm{i}=1,2, \ldots, \mathrm{r}$. Hence $\overline{\Omega_{\tilde{\Lambda}}^{\mathrm{i}}(\lambda)}$ is a finite union of hyper-planes parallel to $\partial \mathrm{H}_{\mathrm{i}}$.

Proof: $h_{i}$ is linear, then the left side is included in the right-hand side. Also, the right-hand side is Zariski closure in $\mathrm{M}_{\mathrm{Q}}$. By the Lemma 2.5,we suppose that $\mathrm{h}_{\mathrm{i}}(\mu)=\mathrm{b} \in \mathrm{h}_{\mathrm{i}}(\mathrm{M})$ with $0 \leq \mathrm{h}_{\mathrm{i}}(\mu)<-\mathrm{h}_{\mathrm{i}}(\lambda)$. Let $\Lambda_{\mathrm{b}}=\left\{\lambda \in \Lambda \vdots \mathrm{h}_{\mathrm{i}}(\mu)=\mathrm{b}\right\}$ with $\Lambda_{\mathrm{b}} \neq \phi . \partial \mathrm{H}_{\mathrm{i}}$ is a face of $\sigma$, then $\operatorname{dim}_{\mathrm{Q}} \mathrm{Q} \Lambda_{0}=\mathrm{r}-1$. Therefore, by the Lemma 2.5, $\overline{\Lambda_{\mathrm{b}}}=\left\{\lambda \in \mathrm{M}_{\mathrm{Q}} \vdots \mathrm{h}_{\mathrm{i}}(\lambda)=\mathrm{b}\right\}$ and $\mu \in \Omega_{\tilde{\Lambda}}^{\mathrm{i}}(\lambda)$.

\section{Proposition 2.7:}

(1) $\mathrm{W} \subseteq \mathrm{D}_{1}(\mathrm{x})$;

(2) $\mathrm{D}_{1}(\mathrm{x})=\underset{\lambda \in \mathrm{M}}{\oplus} \mathrm{X}^{\lambda} \mathrm{I}\left(\overline{\Omega_{\tilde{\Lambda}}(\lambda)}\right)$;

(3) $\overline{\Omega_{\tilde{\Lambda}}(\lambda)}$ is a finite union of hyper-planes each parallel to some $\partial \mathrm{H}_{\mathrm{i}}$

(4) $\Omega_{\tilde{\Lambda}}(\lambda)=\overline{\Omega_{\tilde{\Lambda}}(\lambda)} \cap \tilde{\Lambda}$.

Proof: Clearly the first and the second properties are satisfied by [2]. For the third property, we have $\Omega(\lambda)=\cup \Omega^{\mathrm{i}}(\lambda)$. By the proposition 2.6, $\overline{\Omega_{\widetilde{\Lambda}}^{\mathrm{i}}(\lambda)}$ is a finite union of hyper-planes parallel to $\partial \mathrm{H}_{\mathrm{i}}$, then $\overline{\Omega(\lambda)}=\overline{\cup \Omega^{\mathrm{i}}(\lambda)}=\overline{\cup \Omega^{\mathrm{i}}(\lambda)}$,

and $\overline{\Omega(\lambda)}$ is a finite union of hyper-planes parallel to $\partial \mathrm{H}_{\mathrm{i}}$. For the fourth property, it's clear that $\quad \Omega_{\tilde{\Lambda}}(\lambda) \subseteq \overline{\Omega_{\tilde{\Lambda}}(\lambda)} \cap \tilde{\Lambda}$.

Now we suppose that $\mathrm{F} \subseteq \overline{\Omega_{\tilde{\Lambda}}(\lambda)}$ is a finite union of hyper-planes parallel to some $\partial \mathrm{H}_{\mathrm{i}}$. By [3, lemma 3.5] we have $\lambda+\mathrm{F} \subseteq \mathrm{H}_{\mathrm{i}}^{\mathrm{c}} \Rightarrow \lambda+(\mathrm{F} \cap \tilde{\Lambda}) \subseteq \mathrm{H}_{\mathrm{i}}^{\mathrm{c}}$ 


\section{$\Rightarrow \mathrm{F} \cap \tilde{\Lambda} \subseteq \Omega(\lambda)$}

and

$$
\begin{aligned}
\overline{\Omega_{\tilde{\Lambda}}} \cap \tilde{\Lambda} & =(\cup F) \cap \tilde{\Lambda} \\
& =\cup(\mathrm{F} \cap \tilde{\Lambda}) \\
& \subseteq \Omega_{\tilde{\Lambda}}(\lambda) .
\end{aligned}
$$

Then $\Omega_{\tilde{\Lambda}}(\lambda)=\overline{\Omega_{\tilde{\Lambda}}(\lambda)} \cap \tilde{\Lambda}$.

\section{The new relations:}

In this section, we start with a single relation of Jones and we use this relation to obtain a new general basis.

Proposition 3.1: For $\lambda \in \mathrm{M}=\mathrm{Z} \Lambda=\mathrm{Z} \tilde{\Lambda}$, the following holds:

$$
\begin{aligned}
& \text { (1) } \overline{\Omega_{\tilde{\Lambda}}(\lambda)}=\overline{\Omega_{\Lambda, \tilde{\Lambda}}(\lambda)} \cap\left(\cup_{\mathrm{F} \in \mathrm{Y}} \mathrm{F}\right) \text { where } \mathrm{Y}=\left\{\mathrm{F} \in \mathrm{F}_{1}: \mathrm{F} \subseteq \overline{\Omega_{\tilde{\Lambda}}(\lambda)}\right\} . \\
& \overline{\Omega_{\tilde{\Lambda}, \Lambda}(\lambda)}=\overline{\Omega_{\tilde{\Lambda}}(\lambda)} \cup \underset{\mathrm{F} \in \Sigma}{ }(\mathrm{F}-\lambda) \text { where } \Sigma=\left\{\mathrm{F} \in \mathrm{F}_{1}:(\mathrm{F}-\lambda) \cap \tilde{\Lambda} \neq \phi\right\} .
\end{aligned}
$$

\section{Proof:}

(1) Let $\Phi$ be the set of hyper-plane, then

$$
\overline{\Omega_{\tilde{\Lambda}}(\lambda)}=\cup_{\mathrm{F} \in \Phi} \mathrm{F}=\cup_{\mathrm{F} \in \mathrm{Y}} \mathrm{F} \cup \bigcup_{\mathrm{F} \in \Phi \backslash \mathrm{Y}} \mathrm{F} \text {. }
$$

Now, by the definition $\Omega_{\Lambda, \tilde{\Lambda}}(\lambda)=\{\mu \in \Lambda: \lambda+\mu \notin \tilde{\Lambda}\}$, then

$$
\Omega_{\Lambda, \tilde{\Lambda}}(\lambda)=\Omega_{\tilde{\Lambda}}(\lambda) \cap \Lambda \text {. }
$$

$$
\begin{aligned}
& \text { Also } \overline{\Omega_{\Lambda, \tilde{\Lambda}}(\lambda)}=\overline{\Omega_{\tilde{\Lambda}}(\lambda) \cap \Lambda} \\
& =\overline{\overline{\Omega_{\tilde{\Lambda}}(\lambda)} \cap \tilde{\Lambda} \cap \Lambda} \text {; by (2.7.4) } \\
& =\overline{\overline{\Omega_{\tilde{\Lambda}}(\lambda)} \cap \Lambda} ;(\Lambda \subseteq \tilde{\Lambda}) \\
& =\overline{\left(\underset{F \in \Phi \backslash Y}{\cup} \mathrm{F} \cup \bigcup_{\mathrm{F} \in \mathrm{Y}}\right) \cap \Lambda} \\
& =\overline{\left(\bigcup_{F \in \Phi \backslash Y} F \cap \Lambda\right) \cup\left(\bigcup_{F \in \Phi \backslash Y} F \cap \Lambda\right)} \\
& =\overline{\bigcup_{F \in \phi \backslash Y} F \cap \Lambda} \\
& =\underset{\mathrm{F} \in \phi \backslash \mathrm{Y}}{\cup} \mathrm{F}
\end{aligned}
$$

Thus $\overline{\Omega_{\tilde{\Lambda}}(\lambda)}=\overline{\Omega_{\Lambda, \tilde{\Lambda}}(\lambda)} \cap \underset{\mathrm{F} \in \mathrm{Y}}{\cup} \mathrm{F}$. 
(2) See [3, Proposition 3.11.2] proof.

Jones in [3] gave the following lemma, which is given here with a new

Lemma 3.2: For $\lambda \in \mathrm{M}, \overline{\Omega_{\Lambda}(\lambda)}=\overline{\Omega_{\Lambda, \tilde{\Lambda}}(\lambda)} \cup \bigcup_{\mathrm{F} \in \Sigma^{\prime}}(\mathrm{F}-\lambda)$ where $\Sigma^{\prime}=\left\{\mathrm{F} \in \mathrm{F}_{1}:(\mathrm{F}-\lambda) \cap \Lambda \neq \phi\right\}$

Proof: We have, $\Omega_{\widetilde{\Lambda}}(\lambda)=\Omega_{\widetilde{\Lambda}, \Lambda}(\lambda) \cap \Lambda$. By proposition (3.1):

$$
\begin{aligned}
\Omega_{\Lambda}(\lambda) & =\left(\Omega_{\tilde{\Lambda}}(\lambda) \cup \bigcup_{\mathrm{F} \in \Sigma}(\mathrm{F}-\lambda)\right) \cap \Lambda \\
& =\left(\Omega_{\tilde{\Lambda}}(\lambda) \cap \Lambda\right) \cup\left(\bigcup_{\mathrm{F} \in \Sigma^{\prime}}(\mathrm{F}-\lambda)\right) \\
& =\Omega_{\Lambda, \tilde{\Lambda}}(\lambda) \cup \underset{\mathrm{F} \in \Sigma^{\prime}}{\cup}(\mathrm{F}-\lambda)
\end{aligned}
$$

From Proposition (2.7) and (3.1) and Lemma 3.2, we can write and prove the following:

\section{Theorem 3.3:}

$$
\begin{aligned}
& \text { (1) } \Omega_{\Lambda}(\lambda) \cap \Omega_{\tilde{\Lambda}}(\lambda)=\Omega_{\Lambda, \tilde{\Lambda}}(\lambda) ; \\
& \text { (2) } \Omega_{\Lambda}(\lambda) \cap \tilde{\Lambda}=\Omega_{\Lambda}(\lambda) ; \\
& \text { (3) } \Omega_{\tilde{\Lambda}, \Lambda}(\lambda) \cap \tilde{\Lambda}=\Omega_{\tilde{\Lambda}, \Lambda}(\lambda) ; \\
& \text { (4) } \Omega_{\Lambda, \tilde{\Lambda}}(\lambda) \cap \tilde{\Lambda}=\Omega_{\Lambda, \tilde{\Lambda}}(\lambda) ; \\
& \text { (5) } \Omega_{\Lambda}(\lambda) \cap(\tilde{\Lambda} \backslash \Lambda)=\phi ; \\
& \text { (7) } \Omega_{\Lambda, \tilde{\Lambda}}(\lambda) \cap(\tilde{\Lambda} \backslash \Lambda)=\phi ; \\
&
\end{aligned}
$$

\section{Proof:}

(1) Since $\Omega_{\Lambda}(\lambda)=\Omega_{\tilde{\Lambda}, \Lambda}(\lambda) \cap \Lambda$ and $\Omega_{\Lambda, \tilde{\Lambda}}(\lambda)=\Omega_{\tilde{\Lambda}}(\lambda) \cap \Lambda$, then by Proposition 3.1 and Lemma 3.2, we have

$$
\begin{aligned}
\Omega_{\Lambda, \tilde{\Lambda}}(\lambda) \subseteq & \Omega_{\Lambda}(\lambda) \subseteq \Omega_{\tilde{\Lambda}, \Lambda}(\lambda) \\
\Omega_{\Lambda, \tilde{\Lambda}}(\lambda) \subseteq & \Omega_{\tilde{\Lambda}}(\lambda) \subseteq \Omega_{\tilde{\Lambda}, \Lambda}(\lambda) \\
& \quad \text { Then } \Omega_{\Lambda}(\lambda) \cap \Omega_{\tilde{\Lambda}}(\lambda)=\left(\Omega_{\tilde{\Lambda}, \Lambda}(\lambda) \cap \Lambda\right) \cap \Omega_{\tilde{\Lambda}}(\lambda)
\end{aligned}
$$




$$
\begin{aligned}
& =\Omega_{\tilde{\Lambda}, \Lambda}(\lambda) \cap\left(\Omega_{\tilde{\Lambda}}(\lambda) \cap \Lambda\right) \\
& =\Omega_{\tilde{\Lambda}, \Lambda}(\lambda) \cap \Omega_{\Lambda, \tilde{\Lambda}}(\lambda) \\
& =\Omega_{\Lambda, \tilde{\Lambda}}(\lambda) .
\end{aligned}
$$

(2) $\Omega_{\Lambda}(\lambda) \cap \tilde{\Lambda}=\Omega_{\tilde{\Lambda}, \Lambda}(\lambda) \cap \Lambda \cap \tilde{\Lambda}$

$$
=\Omega_{\tilde{\Lambda}, \Lambda}(\lambda) \cap \Lambda=\Omega_{\Lambda}(\lambda) .
$$

(3) $\Omega_{\tilde{\Lambda}, \Lambda}(\lambda) \cap \tilde{\Lambda}=\left(\left(\bigcup_{\mathrm{F} \in \mathrm{F}_{1}}(\mathrm{~F}-\lambda) \cap \tilde{\Lambda}\right) \cup \Omega_{\tilde{\Lambda}}(\lambda)\right) \cap \tilde{\Lambda}$

$$
\begin{aligned}
& =\left(\bigcup_{F \in F_{1}}(F-\lambda) \cap \tilde{\Lambda}\right) \cup\left(\Omega_{\tilde{\Lambda}}(\lambda) \cap \tilde{\Lambda}\right) \\
& =\left(\bigcup_{F \in F_{1}}(F-\lambda) \cap \tilde{\Lambda}\right) \cup \Omega_{\tilde{\Lambda}}(\lambda) \\
& =\Omega_{\tilde{\Lambda}, \Lambda}(\lambda) .
\end{aligned}
$$

(4) $\Omega_{\Lambda, \tilde{\Lambda}}(\lambda) \cap \tilde{\Lambda}=\left(\Omega_{\Lambda}(\lambda) \cap \Omega_{\tilde{\Lambda}}(\lambda)\right) \cap \tilde{\Lambda}$, by (1)

$$
\begin{aligned}
& =\left(\Omega_{\Lambda}(\lambda) \cap \tilde{\Lambda}\right) \cap \Omega_{\tilde{\Lambda}}(\lambda) \\
& =\Omega_{\Lambda}(\lambda) \cap \Omega_{\tilde{\Lambda}}(\lambda), \quad \operatorname{by}(2) \\
& =\Omega_{\Lambda, \tilde{\Lambda}}(\lambda) .
\end{aligned}
$$

(5) $\Omega_{\Lambda}(\lambda) \cap(\tilde{\Lambda} \backslash \Lambda)=\left(\Omega_{\Lambda}(\lambda) \cap \tilde{\Lambda}\right)-\left(\Omega_{\Lambda}(\lambda) \cap \Lambda\right)$

$$
\begin{aligned}
& =\Omega_{\Lambda}(\lambda)-\Omega_{\Lambda}(\lambda), \operatorname{by}(2) \\
& =\phi .
\end{aligned}
$$

(6) $\Omega_{\Lambda, \tilde{\Lambda}}(\lambda) \cap(\tilde{\Lambda} \backslash \Lambda)=\left(\Omega_{\Lambda, \tilde{\Lambda}}(\lambda) \cap \tilde{\Lambda}\right)-\left(\Omega_{\Lambda, \tilde{\Lambda}}(\lambda) \cap \Lambda\right)$

$$
=\phi \text {. } \quad, \quad \text { by }(4)
$$

(7) $\Omega_{\widetilde{\Lambda}, \Lambda}(\lambda) \cap(\tilde{\Lambda} \backslash \Lambda)=\left(\Omega_{\widetilde{\Lambda}, \Lambda}(\lambda) \cap \tilde{\Lambda}\right)-\left(\Omega_{\widetilde{\Lambda}, \Lambda}(\lambda) \cap \Lambda\right)$

$$
\begin{aligned}
& =\Omega_{\tilde{\Lambda}, \Lambda}(\lambda)-\Omega_{\Lambda}(\lambda), \text { by }(3) \\
& =\Omega_{\tilde{\Lambda}, \Lambda}(\lambda) \backslash \Omega_{\Lambda}(\lambda) .
\end{aligned}
$$

We conclude the following theorem: 


\section{Theorem 3.4:}

$$
\begin{aligned}
& \text { (1) } \Omega_{\Lambda}(\lambda) \cap\left(\bigcup_{\mathrm{F} \in \Sigma}(F-\lambda)\right)=\bigcup_{F \in \Sigma^{\prime}}(F-\lambda) . \\
& (2) \Omega_{\Lambda, \tilde{\Lambda}}(\lambda) \cap\left(\bigcup_{F \in \Sigma}(F-\lambda)\right)=\Omega_{\tilde{\Lambda}}(\lambda) \cap\left(\bigcup_{F \in \Sigma^{\prime}}(F-\lambda)\right)
\end{aligned}
$$

\section{Proof:}

$$
\text { (1) } \begin{aligned}
\Omega_{\Lambda}(\lambda) \cap\left(\bigcup_{\mathrm{F} \in \Sigma}\right. & (F-\lambda))=\left(\Omega_{\Lambda, \tilde{\Lambda}}(\lambda) \cup \bigcup_{\mathrm{F} \in \Sigma^{\prime}}(\mathrm{F}-\lambda)\right) \cap\left(\bigcup_{\mathrm{F} \in \Sigma}(\mathrm{F}-\lambda)\right) \\
& \left.=\left(\Omega_{\Lambda, \tilde{\Lambda}}(\lambda) \cap\left(\bigcup_{\mathrm{F} \in \Sigma}(\mathrm{F}-\lambda)\right)\right) \bigcup\left(\bigcup_{\mathrm{F} \in \Sigma^{\prime}}(\mathrm{F}-\lambda) \cap \bigcup_{\mathrm{F} \in \Sigma}(\mathrm{F}-\lambda)\right)\right) \\
& =\left(\Omega_{\Lambda, \tilde{\Lambda}}(\lambda) \cap\left(\bigcup_{\mathrm{F} \in \Sigma}(\mathrm{F}-\lambda)\right)\right) \bigcup\left(\bigcup_{\mathrm{F} \in \Sigma^{\prime}}(\mathrm{F}-\lambda)\right) \\
& \left.=\left(\Omega_{\tilde{\Lambda}}(\lambda) \cap \Lambda \cap\left(\bigcup_{\mathrm{F} \in \Sigma}(\mathrm{F}-\lambda)\right)\right) \bigcup_{\mathrm{F} \in \Sigma^{\prime}}(\mathrm{F}-\lambda)\right) \\
& =\left(\Omega_{\tilde{\Lambda}}(\lambda) \cap\left(\bigcup_{\mathrm{F} \in \Sigma^{\prime}}(\mathrm{F}-\lambda)\right)\right) \bigcup\left(\bigcup_{\mathrm{F} \in \Sigma^{\prime}}(\mathrm{F}-\lambda)\right) \\
& =\bigcup_{\mathrm{F} \in \Sigma^{\prime}}(\mathrm{F}-\lambda) .
\end{aligned}
$$

(2) $\Omega_{\Lambda, \tilde{\Lambda}}(\lambda) \cap\left(\bigcup_{F \in \Sigma}(F-\lambda)\right)=\Omega_{\Lambda, \tilde{\Lambda}}(\lambda) \cap\left(\bigcup_{F \in F_{1}}(F-\lambda) \cap \tilde{\Lambda}\right)$

$$
\begin{aligned}
& =\Omega_{\Lambda, \tilde{\Lambda}}(\lambda) \cap\left(\bigcup_{\mathrm{F} \in \mathrm{F}_{1}}(\mathrm{~F}-\lambda)\right), \quad \text { by }(3.3 .4) \\
& =\left(\Omega_{\tilde{\Lambda}}(\lambda) \cap \Lambda\right) \cap\left(\bigcup_{\mathrm{F} \in \mathrm{F}_{1}}(F-\lambda)\right) \\
& =\Omega_{\tilde{\Lambda}}(\lambda) \cap\left(\bigcup_{\mathrm{F} \in \Sigma^{\prime}}(F-\lambda)\right)
\end{aligned}
$$




\section{REFERENCES}

[1] Danilov, V. I. (1978), "The geometry of toric varieties", Russian Math. Surveys 33, No.2,pp. 97-154.

[2] Jones, A. G. (1993), "Rings of differential operators on toric varieties", Proc. Edinburgh Math. Soc. 37, pp.143-160.

[3] Jones, A. G. (1995), "Some Morita equivalence of rings of differential operators", J. algebra 173, pp.180-199.

[4] McConnell, J. C. and Robson J. C. (1987), "Non-commutative Noetherian Rings", Pure and Applied Mathematics, A wileyInterscience series of texts, Monographs and tracts.

[5] Musson, I. M. (1987), "Rings of differential operators on invariant rings of tori”, Trans. Amer. Math. Soc.303, No.2, pp.805-827.

[6] Oda, T. (1985),“Convex Bodies and Algebraic Geometry-An Introduction to the Theory of Toric Varieties", in "Ergebnisse der Mathematik und ihrer Grenzgebiete," Vol.15, Springer-Verlag, New York.

[7] Smith, S. P. and Stafford, J. T.(1988), "Differential operators on an affine curve", Proc. London Math. Soc.(3), 56, pp.229-259. 